\title{
A NEW SUBCLASS OF MEROMORPHIC FUNCTIONS DEFINED BY RAPID OPERATOR
}

\author{
Venkateswarlu BOLINENI ${ }^{1}$, Thirupathi Reddy PINNINTI ${ }^{2}$, SUJATHA $^{1}$, \\ and Sridevi SETTIPALLI ${ }^{1}$ \\ ${ }^{1}$ Department of Mathematics, GSS, GITAM University, Doddaballapur- 562 163, Bengaluru \\ Rural, Karnataka, INDIA \\ ${ }^{2}$ Department of Mathematics, Kakatiya University,Warangal- 506 009, Telangana, INDIA
}

Abstract. We present and investigate a new subclass of meromorphic univalent functions described by the Rapid operator in this study. Coefficient inequalities is discussed, as well as distortion properties, closure theorems, Hadamard product. After this, integral transforms for the class $\Sigma^{*}(\vartheta, \varrho, \wp, \theta, \mu)$ are obtained.

\section{INTRODUCTION}

Let $\Sigma$ stands for the function class of the form

$$
\aleph(\hbar)=\frac{1}{\hbar}+\sum_{\ell=1}^{\infty} a_{\ell} \hbar^{\ell}, \ell \in N=\{1,2,3, \cdots\}
$$

analytic in the punctured unit disc $\Upsilon^{*}=\{\hbar \in C: 0<|\hbar|<1\}=\Upsilon \backslash\{0\}$.

A function $\aleph \in \Sigma$ given by (1) is said to be meromorphically starlike of order $\varrho$ if it satisfies the following:

$$
\Re\left\{-\left(\frac{\hbar \aleph^{\prime}(\hbar)}{\aleph(\hbar)}\right)\right\}>\varrho,(\hbar \in \Upsilon)
$$

for some $\varrho(0 \leq \varrho<1)$. We say that $\aleph$ is in the class $\Sigma^{*}(\varrho)$ of such functions.

Similarly a function $\aleph \in \Sigma$ given by (1) is said to be meromorphically convex of order $\varrho$ if it satisfies the following:

$$
\Re\left\{-\left(1+\frac{\hbar \aleph^{\prime \prime}(\hbar)}{\aleph^{\prime}(\hbar)}\right)\right\}>\varrho,(\hbar \in \Upsilon)
$$

2020 Mathematics Subject Classification. 30C45.

Keywords and phrases. Meromorphic, starlike, coefficient estimates, integral operator.

凶 bvlmaths@gmail.com-Corresponding author; reddypt2@gmail.com, sujathavaishnavy@gmail.com; siri_settipalli@yahoo.co.in

(D) 0000-0003-3669-350X; 0000-0002-0034-444X; 0000-0002-2109-3328; 0000-0003-1918-6127.

C 2021 Ankara University
Communications Faculty of Sciences University of Ankara-Series A1 Mathematics and Statistics 
for some $\varrho(0 \leq \varrho<1)$. We say that $\aleph$ is in the class $\Sigma_{\ell}(\varrho)$ of such functions.

Akgul [1,2], Miller 8, Pommerenke [9, Royster 10], Aydogan and Sakar [4 5 11 and Venkateswarlu et al. 14, 15, 16 have all studied the class $\Sigma^{*}(\varrho)$ and numerous other subclasses of $\Sigma$ extensively.

For functions $\aleph \in \Sigma$ given by (1) and $g \in \Sigma$ given by

$$
g(\hbar)=\frac{1}{\hbar}+\sum_{\ell=1}^{\infty} b_{\ell} \hbar^{\ell},
$$

we define the Hadamard product of $\aleph$ and $g$ by

$$
(\aleph * g)(\hbar)=\frac{1}{\hbar}+\sum_{\ell=1}^{\infty} a_{\ell} b_{\ell} \hbar^{\ell}
$$

Jung et al. defined the integral operator on normalised analytic functions in 6 and Lashin 7 updated their operator for meromorphic functions in the following manner:

Lemma 1. For $\aleph \in \Sigma$ given by (1), if the operator $S_{\mu}^{\theta}: \Sigma \rightarrow \Sigma$ is defined by

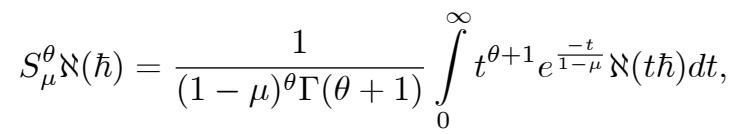

$(0 \leq \mu<1,0 \leq \theta \leq 1$ and $\hbar \in \Upsilon)$ then

$$
S_{\mu}^{\theta} \aleph(\hbar)=\frac{1}{\hbar}+\sum_{\ell=1}^{\infty} \phi_{\ell}(\theta, \mu) a_{\ell} \hbar^{\ell}
$$

where $\phi_{\ell}(\theta, \mu)=(1-\mu)^{\ell+1} \frac{\Gamma(\ell+\theta+2)}{\Gamma(\theta+1)}$ and $\Gamma$ is the familiar Gamma function.

Using the equation (3), it is easily seen that

$$
\hbar\left(S_{\mu}^{\theta} \aleph(\hbar)\right)^{\prime}=\mu S_{\mu}^{\theta-1} \aleph(\hbar)-(\mu+1) S_{\mu}^{\theta} \aleph(\hbar),(0 \leq \mu \leq 1,0 \leq \theta \leq 1) .
$$

We define a new subclass $\Sigma^{*}(\vartheta, \varrho, \wp, \theta, \mu)$ of $\Sigma$ based on Sivaprasad Kumar et al. [13] and Venkateswarlu et al. [14] $\Sigma^{*}(\vartheta, \varrho, \wp, \theta, \mu)$ of $\Sigma$.

Definition 2. For $0 \leq \vartheta<1, \varrho \geq 0,0 \leq \wp<\frac{1}{2}$, we let $\Sigma^{*}(\vartheta, \varrho, \wp, \theta, \mu)$ be the subclass of $\Sigma$ consisting of functions of the form (1) and satisfying the analytic condition

$-\Re\left(\frac{\hbar\left(S_{\mu}^{\theta} \aleph(\hbar)\right)^{\prime 2}\left(S_{\mu}^{\theta} \aleph(\hbar)\right)^{\prime \prime}}{(1-\wp) S_{\mu}^{\theta} \aleph(\hbar)+\wp \hbar\left(S_{\mu}^{\theta} \aleph(\hbar)\right)^{\prime}}+\vartheta\right)>\varrho\left|\frac{\hbar\left(S_{\mu}^{\theta} \aleph(\hbar)\right)^{\prime 2}\left(S_{\mu}^{\theta} \aleph(\hbar)\right)^{\prime \prime}}{(1-\wp) S_{\mu}^{\theta} \aleph(\hbar)+\wp \hbar\left(S_{\mu}^{\theta} \aleph(\hbar)\right)^{\prime}}+1\right|$.

The following lemmas are needed to prove our findings 3 .

Lemma 3. If $\eta$ is a real number and $\omega$ is a complex number then

$$
\Re(\omega) \geq \eta \Leftrightarrow|\omega+(1-\eta)|-|\omega-(1+\eta)| \geq 0 .
$$


Lemma 4. If $\omega$ is a complex number and $\eta, \ell$ are real numbers then

$$
-\Re(\omega) \geq \ell|\omega+1|+\eta \Leftrightarrow-\Re\left(\omega\left(1+\ell e^{i \theta}\right)+\ell e^{i \theta}\right) \geq \eta, \quad(-\pi \leq \theta \leq \pi) .
$$

The key purpose of this paper is to look at some traditional geometric function theory properties for the class of geometric functions, such as coefficient bounds, distortion properties, closure theorems, Hadamard product, and integral transforms.

\section{Coefficient estimates}

We obtain required and adequate conditions for a function $\aleph$ to be in the class in this section.

Theorem 5. Let $\aleph \in \Sigma$ be given by (1). Then $\aleph \in \Sigma^{*}(\vartheta, \varrho, \wp, \theta, \mu)$ iff

$$
\sum_{\ell=1}^{\infty}[(1+(\ell-1) \wp)][\ell(\varrho+1)+(\varrho+\vartheta)] \phi_{\ell}(\theta, \mu) a_{\ell} \leq(1-\vartheta)(1-2 \wp) .
$$

Proof. Let $\aleph \in \Sigma^{*}(\vartheta, \varrho, \wp, \theta, \mu)$. Then by Definition 2 and using Lemma 4 . It suffices to demonstrate that

$$
-\Re\left\{\frac{\hbar\left(S_{\mu}^{\theta} \aleph(\hbar)\right)^{\prime 2}\left(S_{\mu}^{\theta} \aleph(\hbar)\right)^{\prime \prime}}{(1-\wp) S_{\mu}^{\theta} \aleph(\hbar)+\wp \hbar\left(S_{\mu}^{\theta} \aleph(\hbar)\right)^{\prime}}\left(1+\varrho e^{i \theta}\right)+\varrho e^{i \theta}\right\} \geq \vartheta, \quad(-\pi \leq \theta \leq \pi) .
$$

For convenience

$$
\begin{aligned}
C(\hbar)= & -\left[\hbar\left(S_{\mu}^{\theta} \aleph(\hbar)\right)^{\prime 2}\left(S_{\mu}^{\theta} \aleph(\hbar)\right)^{\prime \prime}\right]\left(1+\varrho e^{i \theta}\right) \\
& -\varrho e^{i \theta}\left[(1-\wp) S_{\mu}^{\theta} \aleph(\hbar)+\wp \hbar\left(S_{\mu}^{\theta} \aleph(\hbar)\right)^{\prime}\right] \\
D(\hbar)= & (1-\wp) S_{\mu}^{\theta} \aleph(\hbar)+\wp \hbar\left(S_{\mu}^{\theta} \aleph(\hbar)\right)^{\prime} .
\end{aligned}
$$

That is, the equation (7) is equivalent to

$$
-\Re\left(\frac{C(\hbar)}{D(\hbar)}\right) \geq \vartheta .
$$

We only need to prove that in light of Lemma 3

$$
|C(\hbar)+(1-\vartheta) D(\hbar)|-|C(\hbar)-(1+\vartheta) D(\hbar)| \geq 0 .
$$

Therefore

$$
\begin{aligned}
& |C(\hbar)+(1-\vartheta) D(\hbar)| \\
\geq & (2-\vartheta)(1-2 \wp) \frac{1}{|\hbar|}-\sum_{\ell=1}^{\infty}[\ell-(1-\vartheta)][1+\wp(\ell-1)] \phi_{\ell}(\theta, \mu) a_{\ell}|\hbar|^{\ell} \\
& -\varrho \sum_{\ell=1}^{\infty}(\ell+1)[1+\wp(\ell-1)] \phi_{\ell}(\theta, \mu) a_{\ell}|\hbar|^{\ell}
\end{aligned}
$$

and $|C(\hbar)-(1+\vartheta) D(\hbar)|$ 


$$
\begin{aligned}
\leq & \vartheta(1-2 \wp) \frac{1}{|\hbar|}+\sum_{\ell=1}^{\infty}[\ell+(1+\vartheta)][1+\wp(\ell-1)] \phi_{\ell}(\theta, \mu) a_{\ell}|\hbar|^{\ell} \\
& +\varrho \sum_{\ell=1}^{\infty}(\ell+1)[1+\wp(\ell-1)] \phi_{\ell}(\theta, \mu) a_{\ell}|\hbar|^{\ell} .
\end{aligned}
$$

It is to show that

$$
\begin{aligned}
& |C(\hbar)+(1-\vartheta) D(\hbar)|-|C(\hbar)-(1+\vartheta) D(\hbar)| \\
\geq & 2(1-\vartheta)(1-2 \wp) \frac{1}{|\hbar|}-2 \sum_{\ell=1}^{\infty}[(\ell+\vartheta)(1+(\ell-1) \wp)] \phi_{\ell}(\theta, \mu) a_{\ell}|\hbar|^{\ell} \\
& -2 \varrho \sum_{\ell=1}^{\infty}(\ell+1)(1+(\ell-1) \wp) \phi_{\ell}(\theta, \mu) a_{\ell}|\hbar|^{\ell}
\end{aligned}
$$

$\geq 0$, by the given condition (6).

Conversely suppose $\aleph \in \Sigma^{*}(\vartheta, \varrho, \wp, \theta, \mu)$. Then by Lemma 3 , we have (7).

The inequality (7) is reduced to when the values of $\hbar$ are chosen on the positive real axis

$\Re\left\{\frac{\left[(1-2 \wp)(1-\vartheta)\left(1+\varrho e^{i \theta}\right)\right] \frac{1}{\hbar^{2}}+\sum_{\ell=1}^{\infty}\left\{\ell+\varrho e^{i \theta}(\ell+1)+\vartheta\right\}[1+\wp(\ell-1)] \phi_{\ell}(\theta, \mu) \hbar^{\ell-1}}{(1-2 \wp) \frac{1}{\hbar^{2}}+\sum_{\ell=1}^{\infty}[1+\wp(\ell-1)] \phi_{\ell}(\theta, \mu) a_{\ell} \hbar^{\ell-1}}\right\} \geq 0$.

Since $\Re\left(-e^{i \theta}\right) \geq-\left|e^{i \theta}\right|=-1$, the above inequality is reduced to

$\Re\left\{\frac{\left[(1-2 \wp)(1-\vartheta)\left(1+\varrho e^{i \theta}\right)\right] \frac{1}{r^{2}}+\sum_{\ell=1}^{\infty}\{\ell+\varrho(\ell+1)+\vartheta\}[1+\wp(\ell-1)] \phi_{\ell}(\theta, \mu) a_{\ell} r^{\ell-1}}{(1-2 \wp) \frac{1}{r^{2}}+\sum_{\ell=1}^{\infty}[1+\wp(\ell-1)] \phi_{\ell}(\theta, \mu) r^{\ell-1}}\right\} \geq 0$.

We obtained the inequality (6) by letting $r \rightarrow 1^{-}$and using the mean value theorem.

Corollary 6. If $\aleph \in \Sigma^{*}(\vartheta, \varrho, \wp, \theta, \mu)$ then

$$
a_{\ell} \leq \frac{(1-\vartheta)(1-2 \wp)}{[1+\wp(\ell-1)][\ell(1+\varrho)+(\vartheta+\varrho)] \phi_{\ell}(\theta, \mu)} .
$$

The estimate is sharp for the function

$$
\aleph(\hbar)=\frac{1}{\hbar}+\frac{(1-\vartheta)(1-2 \wp)}{[1+\wp(\ell-1)][\ell(1+\varrho)+(\vartheta+\varrho)] \phi_{\ell}(\theta, \mu)} \hbar^{\ell} .
$$

We get the following corollary by taking $\wp=0$ in Theorem 5 . 
Corollary 7. If $\Sigma^{*}(\vartheta, \varrho, \theta, \mu)$ then

$$
a_{\ell} \leq \frac{1-\vartheta}{[\ell(1+\varrho)+(\vartheta+\varrho)] \phi_{\ell}(\theta, \mu)} .
$$

\section{Distortion theorem}

Theorem 8. If $\aleph \in \Sigma^{*}(\vartheta, \varrho, \wp, \theta, \mu)$ then for $0<|\hbar|=r<1$,

$$
\frac{1}{r}-\frac{(1-\vartheta)(1-2 \wp)}{(2 \varrho+\vartheta+1) \phi_{1}(\theta, \mu)} r \leq|\aleph(\hbar)| \leq \frac{1}{r}+\frac{(1-\vartheta)(1-2 \wp)}{(2 \varrho+\vartheta+1) \phi_{1}(\theta, \mu)} r .
$$

This estimate is sharp for the function

$$
\aleph(\hbar)=\frac{1}{\hbar}+\frac{(1-\vartheta)(1-2 \wp)}{(2 \varrho+\vartheta+1) \phi_{1}(\theta, \mu)} \hbar .
$$

Proof. Since $\aleph(\hbar)=\frac{1}{\hbar}+\sum_{\ell=1}^{\infty} a_{\ell} \hbar^{\ell}$, we have

$$
|\aleph(\hbar)|=\frac{1}{r}+\sum_{\ell=1}^{\infty} a_{\ell} r^{\ell} \leq \frac{1}{r}+r \sum_{\ell=1}^{\infty} a_{\ell} .
$$

Since $\ell \geq 1,(2 \varrho+\vartheta+1) \phi_{1}(\theta, \mu) \leq[1+\wp(\ell-1)][\ell(1+\varrho)+(\varrho+\vartheta)] \phi_{\ell}(\theta, \mu)$, using Theorem 5, we have

$$
\begin{aligned}
(2 \varrho+\vartheta+1) \phi_{1}(\theta, \mu) \sum_{\ell=1}^{\infty} a_{\ell} & \leq \sum_{\ell=1}^{\infty}[1+\wp(\ell-1)][\ell(1+\varrho)+(\varrho+\vartheta)] \phi_{\ell}(\theta, \mu) \\
& \leq(1-\vartheta)(1-2 \wp) \\
\Rightarrow \sum_{\ell=1}^{\infty} a_{\ell} & \leq \frac{(1-\vartheta)(1-2 \wp)}{(2 \varrho+\vartheta+1) \phi_{1}(\theta, \mu)} .
\end{aligned}
$$

Using the above inequality in (13), we have

$$
\begin{aligned}
|\aleph(\hbar)| & \leq \frac{1}{r}+\frac{(1-\vartheta)(1-2 \wp)}{(2 \varrho+\vartheta+1) \phi_{1}(\theta, \mu)} r \\
\text { and }|\aleph(\hbar)| & \geq \frac{1}{r}-\frac{(1-\vartheta)(1-2 \wp)}{(2 \varrho+\vartheta+1) \phi_{1}(\theta, \mu)} r .
\end{aligned}
$$

The estimate is sharp for the function $\aleph(\hbar)=\frac{1}{\hbar}+\frac{(1-\vartheta)(1-2 \wp)}{(2 \varrho+\vartheta+1) \phi_{1}(\theta, \mu)} \hbar$. 8 .

We omit the proof of the following corollary since it is similar to that of Theorem

Corollary 9. If $\aleph \in \Sigma^{*}(\vartheta, \varrho, \wp, \theta, \mu)$ then

$$
\frac{1}{r^{2}}-\frac{(1-\vartheta)(1-2 \wp)}{(2 \varrho+\vartheta+1) \phi_{1}(\theta, \mu)} \leq\left|\aleph^{\prime}(\hbar)\right| \leq \frac{1}{r^{2}}+\frac{((1-\vartheta)(1-2 \wp)}{(2 \varrho+\vartheta+1) \phi_{1}(\theta, \mu)} .
$$

The estimate is sharp for the function given by 12 . 


\section{Closure theorems}

Let the function $\aleph_{j}$ be defined, for $j=1,2, \cdots, m$, by

$$
\aleph_{j}(\hbar)=\frac{1}{\hbar}+\sum_{\ell=1}^{\infty} a_{\ell, j} \hbar^{\ell}, a_{\ell, j} \geq 0 .
$$

Theorem 10. Let the functions $\aleph_{j}, j=1,2, \cdots, m$ defined by (14) be in the class $\Sigma^{*}(\vartheta, \varrho, \wp, \theta, \mu)$. Then the function $h$ defined by

$$
h(\hbar)=\frac{1}{\hbar}+\sum_{\ell=1}^{\infty}\left(\frac{1}{m} \sum_{j=1}^{m} a_{\ell, j}\right) \hbar^{\ell}
$$

also belongs to the class $\Sigma^{*}(\vartheta, \varrho, \wp, \theta, \mu)$.

Proof. Since $\aleph_{j}, j=1,2, \cdots, m$ are in the class $\Sigma^{*}(\vartheta, \varrho, \wp, \theta, \mu)$, it follows from Theorem 5, that

$$
\sum_{\ell=1}^{\infty}[1+\wp(\ell-1)][\ell(1+\varrho)+(\varrho+\vartheta)] \phi_{\ell}(\theta, \mu) a_{\ell, j} \leq(1-\vartheta)(1-2 \wp),
$$

for every $j=1,2, \cdots, m$. Hence

$$
\begin{aligned}
& \sum_{\ell=1}^{\infty}[1+\wp(\ell-1)][\ell(1+\varrho)+(\varrho+\vartheta)] \phi_{\ell}(\theta, \mu)\left(\frac{1}{m} \sum_{j=1}^{m} a_{\ell, j}\right) \\
= & \frac{1}{m} \sum_{j=1}^{m}\left(\sum_{\ell=1}^{\infty}[1+\wp(\ell-1)][\ell(1+\varrho)+(\varrho+\vartheta)] \phi_{\ell}(\theta, \mu) a_{\ell, j}\right) \\
\leq & (1-\vartheta)(1-2 \wp) .
\end{aligned}
$$

From Theorem (6), it follows that $h \in \Sigma^{*}(\vartheta, \varrho, \wp, \theta, \mu)$.

Hence the proof.

Theorem 11. The class $\Sigma^{*}(\vartheta, \varrho, \wp, \theta, \mu)$ is closed under convex linear combinations.

Proof. Let the functions $\aleph_{j}, j=1,2, \cdots, m$ defined by (14) be in the class $\Sigma^{*}(\vartheta, \varrho, \wp, \theta, \mu)$. Then one need only show that function

$$
h(\hbar)=\varsigma \aleph_{1}(\hbar)+(1-\varsigma) \aleph_{2}(\hbar), 0 \leq \varsigma \leq 1
$$

is in the class $\Sigma^{*}(\vartheta, \varrho, \wp, \theta, \mu)$. Since for $0 \leq \varsigma \leq 1$,

$$
h(\hbar)=\frac{1}{\hbar}+\sum_{\ell=1}^{\infty}\left[\varsigma a_{\ell, 1}+(1-\varsigma) a_{\ell, 1}\right] \hbar^{\ell},
$$


with the assistance of the Theorem5, we have

$$
\begin{aligned}
& \sum_{\ell=1}^{\infty}[1+\wp(\ell-1)][\ell(1+\varrho)+(\varrho+\vartheta)] \phi_{\ell}(\theta, \mu)\left[\varsigma a_{\ell, 1}+(1-\varsigma) a_{\ell, 1}\right] \\
\leq & \varsigma(1-\vartheta)(1-2 \wp)+(1-\varsigma)(1-\vartheta)(1-2 \wp) \\
= & (1-\vartheta)(1-2 \wp),
\end{aligned}
$$

which implies that $h \in \Sigma^{*}(\vartheta, \varrho, \wp, \theta, \mu)$.

Theorem 12. Let $\xi \geq 0$. Then $\Sigma^{* \xi}(\vartheta, \varrho, \wp, \theta, \mu) \subseteq N(\varrho, \xi)$, where

$$
\xi=1-\frac{2(1-\vartheta)(1-2 \wp)(1+\varrho)}{(2 \varrho+\vartheta+1)+(1-\vartheta)(1-2 \wp)} .
$$

Proof. If $\aleph \in \Sigma^{* \xi}(\vartheta, \varrho, \wp, \theta, \mu)$ then

$$
\sum_{\ell=1}^{\infty} \frac{[1+\wp(\ell-1)][\ell(1+\varrho)+(\varrho+\vartheta)] \phi_{\ell}(\theta, \mu)}{(1-\vartheta)(1-2 \wp)} a_{\ell} \leq 1 .
$$

We need to find the value of $\xi$ such that

$$
\sum_{\ell=1}^{\infty} \frac{[\ell(1+\varrho)+(\varrho+\xi)] \phi_{\ell}(\theta, \mu)}{1-\xi} a_{\ell} \leq 1 .
$$

Thus it is sufficient to show that

$$
\frac{[\ell(1+\varrho)+(\varrho+\xi)] \phi_{\ell}(\theta, \mu)}{1-\xi} \leq \frac{[1+\wp(\ell-1)][\ell(1+\varrho)+(\varrho+\vartheta)] \phi_{\ell}(\theta, \mu)}{(1-\vartheta)(1-2 \wp)} .
$$

Then

Since

$$
\xi \leq 1-\frac{(\ell+1)(1-\vartheta)(1-2 \wp)(1+\varrho)}{[1+\wp(\ell-1)][\ell(1+\varrho)+(\varrho+\vartheta)]+(1-\vartheta)(1-2 \wp)} .
$$

$$
G(\ell)=1-\frac{(\ell+1)(1-\vartheta)(1-2 \wp)(1+\varrho)}{[1+\wp(\ell-1)][\ell(1+\varrho)+(\varrho+\vartheta)]+(1-\vartheta)(1-2 \wp)}
$$

is an increasing function of $\ell, \ell \geq 1$, we obtain

$$
\xi \leq G(1)=1-\frac{2(1-\vartheta)(1-2 \wp)(1+\varrho)}{(2 \varrho+\vartheta+1)+(1-\vartheta)(1-2 \wp)} .
$$

Theorem 13. Let $\aleph_{0}(\hbar)=\frac{1}{\hbar}$ and

$$
\aleph_{\ell}(\hbar)=\frac{1}{\hbar}+\sum_{\ell=1}^{\infty} \frac{(1-\vartheta)(1-2 \wp)}{[1+\wp(\ell-1)][\ell(1+\varrho)+(\varrho+\vartheta)] \phi_{\ell}(\theta, \mu)} \hbar^{\ell}, \ell \geq 1 .
$$

Then $\aleph$ is in the class $\Sigma^{*}(\vartheta, \varrho, \wp, \theta, \mu)$ iff can be expressed in the form

$$
\aleph(\hbar)=\sum_{\ell=0}^{\infty} \omega_{\ell} \aleph_{\ell}(\hbar),
$$


where $\omega_{\ell} \geq 0$ and $\sum_{\ell=0}^{\infty} \omega_{\ell}=1$

Proof. Assume that

$$
\begin{aligned}
\aleph(\hbar) & =\sum_{\ell=0}^{\infty} \omega_{\ell} \aleph_{\ell}(\hbar) \\
& =\frac{1}{\hbar}+\sum_{\ell=1}^{\infty} \frac{(1-\vartheta)(1-2 \wp)}{[1+\wp(\ell-1)][\ell(1+\varrho)+(\varrho+\vartheta)] \phi_{\ell}(\theta, \mu)} \hbar^{\ell} .
\end{aligned}
$$

Then it follows that

$$
\begin{aligned}
& \sum_{\ell=1}^{\infty} \frac{[1+\wp(\ell-1)][\ell(1+\varrho)+(\varrho+\vartheta)] \phi_{\ell}(\theta, \mu)}{(1-\vartheta)(1-2 \wp)} \frac{(1-\vartheta)(1-2 \wp)}{[1+\wp(\ell-1)][\ell(1+\varrho)+(\varrho+\vartheta)] \phi_{\ell}(\theta, \mu)} \hbar^{\ell} \\
& =\sum_{\ell=1}^{\infty} \omega_{\ell}=1-\omega_{0} \leq 1
\end{aligned}
$$

which implies that $\aleph \in \Sigma^{*}(\vartheta, \varrho, \wp, \theta, \mu)$.

On the other side, assume that the function $\aleph$ defined by (1) be in the class $\aleph \in \Sigma^{*}(\vartheta, \varrho, \wp, \theta, \mu)$. Then

$$
a_{\ell} \leq \frac{(1-\vartheta)(1-2 \wp)}{[1+\wp(\ell-1)][\ell(1+\varrho)+(\varrho+\vartheta)] \phi_{\ell}(\theta, \mu)} .
$$

Setting

$$
\omega_{\ell}=\frac{[1+\wp(\ell-1)][\ell(1+\varrho)+(\varrho+\vartheta)] \phi_{\ell}(\theta, \mu)}{(1-\vartheta)(1-2 \wp)} a_{\ell},
$$

where

$$
\omega_{0}=1-\sum_{\ell=0}^{\infty} \omega_{\ell}
$$

$\aleph$ can be expressed in the form $(20)$, as can be shown.

Corollary 14. The extreme points of the class $\Sigma^{*}(\vartheta, \varrho, \wp, \theta, \mu)$ are the functions $\aleph_{0}(\hbar)=\frac{1}{\hbar}$ and

$$
\aleph_{\ell}(\hbar)=\frac{1}{\hbar}+\frac{(1-\vartheta)(1-2 \wp)}{[1+\wp(\ell-1)][\ell(1+\varrho)+(\varrho+\vartheta)] \phi_{\ell}(\theta, \mu)} \hbar^{\ell} .
$$

\section{Modified Hadamard PRODUCTS}

Let the functions $\aleph_{j}(j=1,2)$ defined by (14). The modified Hadamard product of $\aleph_{1}$ and $\aleph_{2}$ is defined by

$$
\left(\aleph_{1} * \aleph_{2}\right)(\hbar)=\frac{1}{\hbar}+\sum_{\ell=1}^{\infty} a_{\ell, 1} a_{\ell, 2} \hbar^{\ell}=\left(\aleph_{2} * \aleph_{1}\right)(\hbar)
$$


Theorem 15. Let the function $\aleph_{j}(j=1,2)$ defined by (14) be in the class $\Sigma^{*}(\vartheta, \varrho, \wp, \theta, \mu)$. Then $\aleph_{1} * \aleph_{2} \in \Sigma^{*}(\vartheta, \varrho, \wp, \theta, \mu)$, where

$$
\varphi=1-\frac{2(1-\vartheta)^{2}(1-2 \wp)(1+\varrho)}{(2 \varrho+\vartheta+1)^{2} \phi_{1}(\theta, \mu)+(1-\vartheta)^{2}(1-2 \wp)} .
$$

The estimate is sharp for the functions $\aleph_{j}(j=1,2)$ given by

$$
\aleph_{j}(\hbar)=\frac{1}{\hbar}+\frac{(1-\vartheta)(1-2 \wp)}{(2 \varrho+\vartheta+1) \phi_{1}(\theta, \mu)} \hbar, \quad(j=1,2) .
$$

Proof. Using the same method that Schild and Silverman 12 used earlier, we need to find the largest real parameter $\varphi$ such that

$$
\sum_{\ell=1}^{\infty} \frac{[1+\wp(\ell-1)][\ell(1+\varrho)+(\varrho+\varphi)] \phi_{\ell}(\theta, \mu)}{(1-\varphi)(1-2 \wp)} a_{\ell, 1} a_{\ell, 2} \leq 1 .
$$

Since $\aleph_{j} \in \Sigma^{*}(\vartheta, \varrho, \wp, \theta, \mu), j=1,2$, we readily see that

$$
\sum_{\ell=1}^{\infty} \frac{[1+\wp(\ell-1)]\left[\ell(1+\varrho)+(\varrho+\vartheta] \phi_{\ell}(\theta, \mu)\right.}{(1-\vartheta)(1-2 \wp)} a_{\ell, 1} \leq 1
$$

and

$$
\sum_{\ell=1}^{\infty} \frac{[1+\wp(\ell-1)]\left[\ell(1+\varrho)+(\varrho+\vartheta] \phi_{\ell}(\theta, \mu)\right.}{(1-\vartheta)(1-2 \wp)} a_{\ell, 2} \leq 1 .
$$

By Cauchy- Schwarz inequality, we have

$$
\sum_{\ell=1}^{\infty} \frac{[1+\wp(\ell-1)]\left[\ell(1+\varrho)+(\varrho+\vartheta] \phi_{\ell}(\theta, \mu)\right.}{(1-\vartheta)(1-2 \wp)} \sqrt{a_{\ell, 1} a_{\ell, 2}} \leq 1 .
$$

Then merely demonstrating that is necessary

$$
\begin{aligned}
& \sum_{\ell=1}^{\infty} \frac{[1+\wp(\ell-1)][\ell(1+\varrho)+(\varrho+\varphi)] \phi_{\ell}(\theta, \mu)}{(1-\varphi)(1-2 \wp)} a_{\ell, 1} a_{\ell, 2} \\
\leq & \sum_{\ell=1}^{\infty} \frac{[1+\wp(\ell-1)]\left[\ell(1+\varrho)+(\varrho+\vartheta] \phi_{\ell}(\theta, \mu)\right.}{(1-\vartheta)(1-2 \wp)} \sqrt{a_{\ell, 1} a_{\ell, 2}}
\end{aligned}
$$

or equivalently that

$$
\sqrt{a_{\ell, 1} a_{\ell, 2}} \leq \frac{[\ell(1+\varrho)+(\varrho+\vartheta](1-\varphi)}{[\ell(1+\varrho)+(\varrho+\varphi](1-\vartheta)} .
$$

Hence, it light of the inequality (26), then merely demonstrating that is necessary

$$
\frac{(1-\vartheta)(1-2 \wp)}{[1+\wp(\ell-1)]\left[\ell(1+\varrho)+(\varrho+\vartheta] \phi_{\ell}(\theta, \mu)\right.} \leq \frac{[\ell(1+\varrho)+(\varrho+\vartheta](1-\varphi)}{[\ell(1+\varrho)+(\varrho+\varphi](1-\vartheta)} .
$$

It follows from 27) that

$$
\varphi \leq 1-\frac{(1-\vartheta)^{2}(1-2 \wp)(1+\varrho)(\ell+1)}{[1+\wp(\ell-1)]\left[\ell(1+\varrho)+(\varrho+\vartheta]^{2} \phi_{\ell}(\theta, \mu)+(1-\vartheta)^{2}(1-2 \wp)\right.} .
$$


Now defining the function $E(\ell)$,

$$
E(\ell)=1-\frac{(1-\vartheta)^{2}(1-2 \wp)(1+\varrho)(\ell+1)}{[1+\wp(\ell-1)]\left[\ell(1+\varrho)+(\varrho+\vartheta]^{2} \phi_{\ell}(\theta, \mu)+(1-\vartheta)^{2}(1-2 \wp)\right.} .
$$

We see that $E(\ell)$ is an increasing of $\ell, \ell \geq 1$. Therefore, we conclude that

$$
\varphi \leq E(\ell)=1-\frac{2(1-\vartheta)^{2}(1-2 \wp)(1+\varrho)}{(2 \varrho+\vartheta+1)^{2} \phi_{1}(\theta, \mu)+(1-\vartheta)^{2}(1-2 \wp)},
$$

Hence the proof.

The following theorem is obtained using arguments close to those used in the proof of 15 ,

Theorem 16. Let the function $\aleph_{1}$ defined by (14) be in the class $\Sigma^{*}(\vartheta, \varrho, \wp, \theta, \mu)$. Suppose also that the function $\aleph_{2}$ defined by (14) be in the class $\Sigma^{*}(\rho, \vartheta, \varrho, \wp, \theta, \mu)$. Then $\aleph_{1} * \aleph_{2} \in \Sigma^{*}(\zeta, \vartheta, \varrho, \wp, \theta, \mu)$, where

$$
\zeta=1-\frac{2(1-\vartheta)(1-\rho)(1-2 \wp)(1+\varrho)}{(2 \varrho+\vartheta+1)(2 \varrho+\rho+1) \phi_{1}(\theta, \mu)+(1-\vartheta)(1-\rho)(1-2 \wp)} .
$$

The estimate is sharp for the functions $\aleph_{j}(j=1,2)$ given by

$$
\aleph_{1}(\hbar)=\frac{1}{\hbar}+\frac{(1-\vartheta)(1-2 \wp)}{(2 \varrho+\vartheta+1) \phi_{1}(\theta, \mu)} \hbar
$$

and

$$
\aleph_{2}(\hbar)=\frac{1}{\hbar}+\frac{(1-\rho)(1-2 \wp)}{(2 \varrho+\rho+1) \phi_{1}(\theta, \mu)} \hbar .
$$

Theorem 17. Let the function $\aleph_{j}(j=1,2)$ defined by 14 be in the class $\Sigma^{*}(\vartheta, \varrho, \wp, \theta, \mu)$. Then the function

$$
h(\hbar)=\frac{1}{\hbar}+\sum_{\ell=1}^{\infty}\left(a_{\ell, 1}^{2}+a_{\ell, 2}^{2}\right) \hbar^{\ell}
$$

belongs to the class $\Sigma^{*}(\varepsilon, \vartheta, \varrho, \wp, \theta, \mu)$, where

$$
\varepsilon=1-\frac{4(1-\vartheta)^{2}(1-2 \wp)(1+\varrho)}{(2 \varrho+\vartheta+1)^{2} \phi_{1}(\theta, \mu)+2(1-\vartheta)^{2}(1-2 \wp)} .
$$

The estimate is sharp for the functions $\aleph_{j}(j=1,2)$ given by (24).

Proof. By using Theorem 5 we obtain

$$
\begin{aligned}
& \sum_{\ell=1}^{\infty}\left\{\frac{[1+\wp(\ell-1)][\ell(1+\varrho)+(\varrho+\vartheta)] \phi_{\ell}(\theta, \mu)}{(1-\vartheta)(1-2 \wp)}\right\}^{2} a_{\ell, 1}^{2} \\
\leq & \sum_{\ell=1}^{\infty}\left\{\frac{[1+\wp(\ell-1)][\ell(1+\varrho)+(\varrho+\vartheta)] \phi_{\ell}(\theta, \mu)}{(1-\vartheta)(1-2 \wp)} a_{\ell, 1}\right\}^{2} \leq 1
\end{aligned}
$$


and

$$
\begin{aligned}
& \sum_{\ell=1}^{\infty}\left\{\frac{[1+\wp(\ell-1)][\ell(1+\varrho)+(\varrho+\vartheta)] \phi_{\ell}(\theta, \mu)}{(1-\vartheta)(1-2 \wp)}\right\}^{2} a_{\ell, 2}^{2} \\
\leq & \sum_{\ell=1}^{\infty}\left\{\frac{[1+\wp(\ell-1)][\ell(1+\varrho)+(\varrho+\vartheta)] \phi_{\ell}(\theta, \mu)}{(1-\vartheta)(1-2 \wp)} a_{\ell, 2}\right\}^{2} \leq 1 .
\end{aligned}
$$

It follows from 31 and 32 that

$$
\sum_{\ell=1}^{\infty} \frac{1}{2}\left\{\frac{[1+\wp(\ell-1)][\ell(1+\varrho)+(\varrho+\vartheta)] \phi_{\ell}(\theta, \mu)}{(1-\vartheta)(1-2 \wp)}\right\}^{2}\left(a_{\ell, 1}^{2}+a_{\ell, 2}^{2}\right) \leq 1 .
$$

Therefore, we need to find the largest $\varepsilon$ such that

$$
\begin{aligned}
& \frac{[1+\wp(\ell-1)][\ell(1+\varrho)+(\varrho+\varepsilon)] \phi_{\ell}(\theta, \mu)}{(1-\varepsilon)(1-2 \wp)} \\
\leq & \frac{1}{2}\left\{\frac{[1+\wp(\ell-1)][\ell(1+\varrho)+(\varrho+\vartheta)] \phi_{\ell}(\theta, \mu)}{(1-\vartheta)(1-2 \wp)}\right\}^{2},
\end{aligned}
$$

that is

$$
\varepsilon \leq 1-\frac{2(1-\vartheta)^{2}(1-2 \wp)(1+\varrho)(\ell+1)}{1+\wp(\ell-1)][\ell(1+\varrho)+(\varrho+\vartheta)]^{2} \phi_{\ell}(\theta, \mu)+2(1-\vartheta)^{2}(1-2 \wp)} .
$$

Since

$$
G(\ell)=1-\frac{2(1-\vartheta)^{2}(1-2 \wp)(1+\varrho)(\ell+1)}{[1+\wp(\ell-1)][\ell(1+\varrho)+(\varrho+\vartheta)]^{2} \phi_{\ell}(\theta, \mu)+2(1-\vartheta)^{2}(1-2 \wp)}
$$

is an increasing function of $\ell, \ell \geq 1$, we obtain

$$
\varepsilon \leq G(1)=\frac{4(1-\vartheta)^{2}(1-2 \wp)(1+\varrho)}{(2 \varrho+\vartheta+1)^{2} \phi_{1}(\theta, \mu)+2(1-\vartheta)^{2}(1-2 \wp)}
$$

and hence the proof.

\section{Integral operators}

Theorem 18. Let the functions $\aleph$ given by (1) be in the class $\Sigma^{*}(\vartheta, \varrho, \wp, \theta, \mu)$. Then the integral operator

$$
F(\hbar)=c \int_{0}^{1} u^{c} \aleph(u \hbar) d u, 0<u \leq 1, c>0
$$

is in the class $\Sigma^{*}(\vartheta, \varrho, \wp, \theta, \mu)$, where

$$
\xi=1-\frac{2 c(1-\vartheta)(1+\varrho)}{(c+2)(2 \varrho+\vartheta+1)+c(1-\vartheta)} .
$$

The estimate is sharp for the function $\aleph$ given by 12 . 
Proof. Let $\aleph \in \Sigma^{*}(\vartheta, \varrho, \wp, \theta, \mu)$. Then

$$
\begin{aligned}
F(\hbar) & =c \int_{0}^{1} u^{c} \aleph(u \hbar) d u \\
& =\frac{1}{\hbar}+\sum_{\ell=1}^{\infty} \frac{c}{\ell+c+1} a_{\ell} \hbar^{\ell} .
\end{aligned}
$$

Thus it is enough to show that

$$
\sum_{\ell=1}^{\infty} \frac{c[1+\wp(\ell-1)][\ell(1+\varrho)+(\varrho+\xi)] \phi_{\ell}(\theta, \mu)}{(\ell+c+1)(1-\xi)(1-2 \wp)} a_{\ell} \leq 1 .
$$

Since $\aleph \in \Sigma^{*}(\vartheta, \varrho, \wp, \theta, \mu)$, then

$$
\sum_{\ell=1}^{\infty} \frac{[1+\wp(\ell-1)][\ell(1+\varrho)+(\varrho+\vartheta)] \phi_{\ell}(\theta, \mu)}{(1-\vartheta)(1-2 \wp)} a_{\ell} \leq 1 .
$$

From $(35)$ and $(36)$, we have

$$
\frac{[\ell(1+\varrho)+(\varrho+\xi)]}{(\ell+c+1)(1-\xi)} \leq \frac{[\ell(1+\varrho)+(\varrho+\vartheta)]}{(1-\vartheta)} .
$$

Then

$$
\xi \leq 1-\frac{c(1-\vartheta)(\ell+1)(1+\varrho)}{(\ell+c+1)[\ell(1+\varrho)+(\varrho+\vartheta)]+c(1-\vartheta)} .
$$

Since

$$
Y(\ell)=1-\frac{c(1-\vartheta)(\ell+1)(1+\varrho)}{(\ell+c+1)[\ell(1+\varrho)+(\varrho+\vartheta)]+c(1-\vartheta)}
$$

is an increasing function of $\ell, \ell \geq 1$, we obtain

$$
\xi \leq Y(1)=1-\frac{2 c(1-\vartheta)(1+\varrho)}{(c+2)(2 \varrho+\vartheta+1)+c(1-\vartheta)}
$$

and hence the proof.

\section{Conclusion}

This research has introduced a new subclass of meromorphic functions defined by Rapid operator and studied some basic properties of geometric function theory. Accordingly, some results to coefficient estimates, distortion properties, closure theorems, hadamard product and integral transforms have been considered, inviting further research for this field of study.

Author Contribution Statements All authors contributed equally to the design and implementation of the research, to the analysis of the results and to the writing of the manuscript. 
Declaration of Competing Interests The authors declare that there is no conflict of interest regarding the publication of this.

Acknowledgments The authors are thankful to the editor and referee(s) for their valuable comments and suggestions which helped very much in improving the paper.

\section{REFERENCES}

[1] Akgul, A., A certain subclass of meromorphic functions with positive coefficients associated with an integral operator, Honam Math. J., 39(3) (2017), 331-347. https://doi.org/10.1016/j.mcm.2010.12.051

[2] Akgul, A., A new subclass of meromorphic functions with positive and fixed second coefficients defined by the Rapid-Operator, Commun. Fac. Sci. Univ. Ank. Series-A1, 66(2) (2017), 113. DOI: $10.1501 /$ Commua1_0000000796

[3] Aqlan, E., Jhangiri, J. M., Kulkarni, S. R., Class of k-uniformly convex and starlike functions, Tamkang, J. Math., 35 (2004), 261-266. https://doi.org/10.5556/j.tkjm.35.2004.207

[4] Aydogan, S. M., Sakar, F. M., Radius of starlikeness of p-valent lambda fractional operator, Applied Mathematics and Computation, 357 (2019), 374-378. https://doi.org/10.1016/j.amc.2018.11.067

[5] Aydogan, S. M., Sakar, F. M., On convex functions with complex order through bounded boundary rotation, Mathematics in Computer Science, 13 (2019), 433-439. https://doi.org/10.1007/s11786-019-00405-8

[6] Jung, I. S., Kim, Y. C., Srivastava, H. M., The Hardy spaces of analytic functions associated with certain one parameter families of integral operators, J. Math. Anal. Appl., 176(1) (1993), 138-147. https://doi.org/10.1006/jmaa.1993.1204

[7] Lashin, A. Y., On certain subclasses of meromorphic functions associated with certain integral operators, Comput. Math. Appl., 59(1) (2010), 524-531. https://doi.org/10.1016/j.camwa.2009.06.015

[8] Miller, J. E., Convex meromorphic mapping and related functions, Proc. Amer. Math. Soc., 25 (1970), 220-228. https://doi.org/10.1090/S0002-9939-1970-0259098-7

[9] Pommerenke, C., On meromorphic starlike functions, Pacific J. Math., 13 (1963), 221-235. DOI: $10.2140 /$ pjm.1963.13.221

[10] Royster, W. C., Meromorphic starlike univalent functions, Trans. Amer. Math. Soc., 107 (1963), 300-308. https://doi.org/10.1090/S0002-9947-1963-0148895-5

[11] Sakar, F. M., Estimating coefficients for certain subclasses of meromorphic and bi-univalent functions, J. of Ineq. and Appl., 283 (2018), 8 pages. https://doi.org/10.1186/s13660-018$1879-4$

[12] Schild, A., Silverman, H., Convolutions of univalent functions with negative coefficients, Ann. Univ. Mariae Curie-Sklodowska Sect. A, 29 (1975), 99-107.

[13] Sivaprasad Kumar, S., Ravichandran, V., Murugusundaramoorthy, G., Classes of meromorphic p-valent parabolic starlike functions with positive coefficients, Aust. J. Math. Anal. Appl., 2(2) (2005), art. 3, 1-9.

[14] Venkateswarlu, B., Thirupathi Reddy, P., Rani, N., Certain subclass of meromorphically uniformly convex functions with positive coefficients, Mathematica (Cluj), 61(84) (1) (2019), 85-97. DOI: 10.24193/mathcluj.2019.1.08

[15] Venkateswarlu, B., Thirupathi Reddy, P., Rani, N., On new subclass of meromorphically convex functions with positive coefficients, Surveys in Math. and its Appl., 14 (2019), 49-60.

[16] Venkateswarlu, B., Thirupathi Reddy, P., Meng, C., Madhuri Shilpa, R., A new subclass of meromorphic functions with positive coefficients defined by Bessel function, Note di Math., 40(1) (2020), 13-25. DOI: 10.1285/i15900932v40n1p13 